\section{EPV172/\#118 OLAPARIB SINGLE- CENTRE EXPERIENCE IN RELAPSED EPITHELIAL OVARIAN CANCER IN SLOVENIA}

B Gregorič ${ }^{*}$, E Škof. Institute of Oncology Ljubljana, Medical Oncology Department, Ljubljana, Slovenia

\subsection{6/ijgc-2021-IGCS.243}

Objectives To evaluate the safety and efficacy of olaparib in treatment of patients with BRCA $1 / 2$ mutated relapsed epithelial ovarian cancer.

Methods Retrospective analysis of patients with BRCA 1/2 mutated relapsed epithelial ovarian cancer treated with olaparib at the Institute of Oncology Ljubljana in the period from Nov 2015 to Dec 2020.

Results In the observed period, a total of 88 patients with BRCA 1/2 mutated relapsed epithelial ovarian cancer were treated with olaparib. Median age of patients was 60 years. Majority of patients $(61 \%)$ had 1 st relapse of the disease. Majority of patients (74\%) had germline BRCA 1 gene mutation. Majority of patients $(85 \%)$ had at least one adverse event during olaparib treatment. The most common adverse events (all grades) were: nausea (59\%), fatigue (59\%), anemia (25\%), dispepsia (14\%), diarrhea (11\%), dysgeusia (10\%), neutropenia (6\%) and arrhythmia (1\%). Severe adverse events (grade $3 / 4$ ) had $10 \%$ of patients: anemia 9\%, nausea 1\%. Median follow up was 40 months. Median PFS was 14,3 months, median OS was 20,4 months. PFS was in correlation to the type of BRCA gene mutation: $80 \%$ of patients with somatic BRCA $1 / 2$ gene mutation were progression-free, $55 \%$ of patients with germline BRCA 2 gene mutation were progression-free, while $32 \%$ of patients with germline BRCA 1 gene mutation were progression-free $(p=0,021)$. The type fo BRCA $1 / 2$ gene mutation did not correlate with OS.

Conclusions Analysis shows olaparib is safe and effective maintainance treatment in BRCA1/2 relapsed epithelial ovarian cancer with results that a comparable to those published in Study19 and SOLO-2.

\section{EPV173/\#134 THE CORRELATION BETWEEN BRCA STATUS AND SURGICAL CYTOREDUCTION IN HIGH-GRADE SEROUS OVARIAN CARCINOMA}

${ }^{1}$ RS Kim*, ${ }^{2} \mathrm{~J}$ Malcolmson, ${ }^{3} \mathrm{X}$ Li, ${ }^{1} \mathrm{M}$ Bernardini, 'L Hogen, ${ }^{1} \mathrm{~T}$ May. ${ }^{1}$ Princess Margaret Cancer Centre/University of Health Network/Sinai Health Systems, Gynecologic Oncology, Toronto, Canada; 'University Health Network, Familial Cancer Clinic, Toronto, Canada; ${ }^{3}$ Princess Margaret Cancer Centre, Biostatistics, Toronto, Canada

10.1136/ijgc-2021-IGCS.244

Objectives The aim of this study is to compare the amount of residual disease following primary cytoreductive surgery (PCS) in BRCA-mutated (BRCAm) and wildtype (BRCAwt) highgrade serous ovarian cancer (HGSC), and to assess whether BRCA status is an independent predictor of residual disease.

Methods We conducted a retrospective analysis of patients with stage III/IV HGSC with known germline and somatic BRCA status, treated with PCS from 2000 to 2017. We compared the cytoreduction outcomes and built a predictive model to assess whether BRCA status was associated with amount of residual disease at the time of PCS.

Results of 303 women, 120 harbored germline or somatic BRCA mutations (40\%) and 183 were BRCAwt (60\%).
BRCAm women tended to be younger (54 vs. 59; p <0.001), but there were no differences between the two groups in disease burden at presentation, surgical complexity, length of surgery, or perioperative complications. The BRCAm group had a higher rate of complete cytoreduction to no residual disease (0mm) [72\% vs. 48\%], and a lower rate of optimal cytoreduction $(1-9 \mathrm{~mm})$ [19\% vs. $38 \%]$ or suboptimal cytoreduction $(\geq 10 \mathrm{~mm}) \quad[10 \%$ vs. $14 \%] \quad(\mathrm{p}<0.001)$. After accounting for length of surgery, CA-125 level, disease scores and surgical complexity scores, BRCAm status was predictive of complete cytoreduction to $0 \mathrm{~mm}$ residual disease (OR 5.2; 95\% CI 2.44-11.1; $\mathrm{p}<0.001)$.

Conclusions BRCAm status is predictive of complete cytoreduction at time of PCS in HGSC. Timely availability of BRCA testing is paramount as it may aid in the therapeutic decision making between PCS or neoadjuvant chemotherapy in women with newly diagnosed HGSC.

\section{EPV174/\#144 PERIOPERATIVE BLOOD MANAGEMENT OF JEHOVAH'S WITNESSES UNDERGOING CYTOREDUCTIVE SURGERY FOR ADVANCED OVARIAN CANCER}

II Palaia, 'G Caruso, ${ }^{1} V$ Di Donato*, ${ }^{1} G$ Perniola, ${ }^{2} G$ Ferrazza, ${ }^{2} E$ Panzini, ${ }^{1} M$ Scudo, ${ }^{1} \mathrm{R}$ Gallo, ${ }^{3} \mathrm{~L}$ Muzii, ${ }^{1} \mathrm{P}$ Benedetti Panici. 'Sapienza University of Rome, Department of Maternal and Child Health and Urological Sciences, Rome, Italy; ${ }^{2}$ Sapienza University of Rome, Department of Immunohematology and Transfusion Medicine, Rome, Italy; ${ }^{3}$ Sapienza University, Gynecologic Oncology, Rome, Italy

\subsection{6/ijgc-2021-IGCS.245}

Objectives This study aimed to evaluate the efficacy and feasibility of a perioperative Bloodless Medicine and Surgery (BMS) protocol in reducing severe postoperative anemia $(\mathrm{Hb}<7 \mathrm{~g} / \mathrm{dL})$ in Jehovah's Witnesses (JWs) undergoing cytoreductive surgery for advanced epithelial ovarian cancer (AEOC).

Methods This was a single-institution retrospective study enrolling JWs who underwent elective bloodless surgery for AEOC between October 2017 and April 2020. All patients followed a standardized BMS protocol based on ferric carboxymaltose (FCM) and erythropoietin (EPO) if indicated.

Results Twenty-five patients with a mean age of 61.7 years (range, 35-80) were enrolled. Preoperatively, 10 patients (40\%) were mildly anemic (mean $\mathrm{Hb}$ of $10.2 \mathrm{~g} / \mathrm{dL}$ [range, 9.2-11.4]) and received FCM. Only 4 (16\%) patients had severe anemia after surgery (mean $\mathrm{Hb}$ of $6.1 \mathrm{~g} / \mathrm{dL}$ [range, 4.1-6.9]) and received FCM and EPO. Compared to patients with postoperative $\mathrm{Hb}>7 \mathrm{~g} / \mathrm{dL}$, those with $\mathrm{Hb}<7 \mathrm{~g} / \mathrm{dL}$ reported higher mean BMI $\left(25.8 \pm 1.8\right.$ vs $30.7 \pm 1.8 \mathrm{~kg} / \mathrm{m}^{2}$; $\mathrm{p}<0.001)$, mean baseline CA125 $(236.1 \pm 184.5$ vs 783.7 $\pm 273.5 \mathrm{IU} / \mathrm{mL} ; \mathrm{p}<0.001)$, median surgical complexity score ( 2 vs $10 ; \mathrm{p}<0.001$ ), and postoperative overall complications $(100 \%$ vs $14.3 \%$; $<0.001)$. Moreover, these patients showed longer mean operating time $(3.4 \pm 0.6$ vs $5.5 \pm 0.4 \mathrm{~h}$; $\mathrm{p}<0.001)$, hospital length of stay $(5.5 \pm 0.7$ vs $24.0 \pm 9.8$ days; $\mathrm{p}<0.001)$, and time to adjuvant chemotherapy $(27.2 \pm 2.6$ vs $65.3 \pm 13.4$ days; $\mathrm{p}<0.001)$.

Conclusions The use of a multidisciplinary BMS protocol is safe and effective in reducing the rate of severe postoperative anemia and improving surgical and oncological outcomes of JWs with AEOC. Further large-scale, prospective studies are required to confirm these data. 